\title{
A RESPIRATORY STIMULANT AND TOXIC SUBSTANCE EXTRACTABLE FROM LUNG TISSUE
}

\author{
WILLIAM B. WhERRY AND D. M. ERVIN \\ From the Laboratories of Bacteriology and Pathology, University of Cincinnati
}

The interesting method we shall describe of producing accelerated respiration or death was discovered accidentally in an experiment with tuberculous tissue from a rabbit's lung. A piece of the tissue, 6 by $8 \mathrm{~mm}$., filled with tubercles, was ground up with sand, suspended in $8 \mathrm{c} \mathrm{c}$ of $0.9 \% \mathrm{NaCl}$ solution, centrifuged to remove coarse particles, and $1 \mathrm{c} \mathrm{c}$ of the supernatant fluid injected intravenously into another rabbit, which immediately fell on its side with marked inspiratory dyspnea, followed by convulsions and opisthotonos, and died a few seconds later.

We shall not detail the earlier experiments during which we thought we might be dealing with a toxin of the tubercle bacillus. Control experiments soon showed that tuberculosis played no part in the phenomenon.

We soon found that normal rabbit lung extract was not only fatal to rabbits, but also to guinea-pigs, when administered intravenously, though not to the same degree. On the other hand, guinea-pig lung extract is more fatal to guinea-pigs than to rabbits. It was only after a number of injections that we standardized our dose. This was done by weighing a piece of lung of about the same size as we had user? before.

The Crude Lung Extract.-Lung tissue, $0.6 \mathrm{gm}$., was ground to a fine paste with sand in a mortar, and the grinding continued while $6 \mathrm{cc}$ of $0.9 \% \mathrm{NaCl}$ solution was added slowly. The toxicity of the extract was not affected appreciably by the time devoted to grinding, e. g., 3 or 10 minutes. The extract was then centrifuged at 1,2001,500 revolutions a minute and the supernatant fluid pipetted off from below the slight scum which rises to the surface. It did not seem to make much difference whether the centrifugation was carried out only for a few minutes or for an hour or two at the speed mentioned. One c c of the crude extract does not really contain $0.1 \mathrm{gm}$. of lung tissue for most of the tissue remains either in the mortar or in the 
centrifuge tube. Such an extract is deeply tinged with hemoglobin and may be opaque or translucent according to the centrifugation it has been subjected to. The fatal intravenous dose of this rabbit lung extract for rabbits varying from $800-2,000 \mathrm{gm}$. in weight, is $0.3 \mathrm{cc}$. This and larger doses kill in a few seconds; $0.1 \mathrm{c} \mathrm{c}$ fails to kill but markedly accelerates the respiration.

The guinea-pig lung extracts were made in the same way.

Symptoms.-If a rabbit be placed on the floor immediately after inoculation with $0.3 \mathrm{cc}$ into an ear vein it may or may not lope off for a few steps. In a few seconds it becomes perfectly still, draws itself together, its respiration is accelerated; it sways a little from side to side; its respirations cease, and it falls on its side extended at full length. Then it goes into convulsions which may be violent or consist of a few running motions with all the legs, the head is retracted in extreme opisthotonos; the mouth opens; occasionally it cries out; one or more deep inspirations is followed by cessation of respiration. At this time the pupils are widely dilated and the superficial muscles of the body sometimes undergo rapidly repeated contractions, giving the appearance of violent shivering.

When dissected immediately after death, there is little abnormal found. The heart still beats after respiration ceases. There is no particular congestion of any of the organs. We have thought that the brain was abnormally pale. The lungs collapse on opening the thorax. The coagtulability of the blood is delayed - altho we have not made a special study of this point, the delay is sometimes considerable, as shown in Rabbit 25. Intestinal peristalsis continues for some time as usual.

In the case of young rabbits which have received one or two sublethal doses we have twice observed a paralysis of the hind legs developing 3 or 4 days after inoculation (Rabbits 9 and 16). Rabbit 16 showed a noncoagulated hemorrhage into the cord at the level of the 5 th-6th rib with complete softening of the cord. No bacteria were found in smears.

A sublethal dose in young as well as old rabbits accelerates the respiration at once to $30-40$ per 10 seconds, while the normal resting rate is 12-16. There is a transitory but apparent weakness of the hind legs.

The following illustrates the results of inoculation of standard crude extract. 
Rabbits Injected with Extract of Rabbit Lung.-Rabbit 6, 1,960 gm., $1 \mathrm{cc}$ of clear extract centrifuged at 1,500 revolutions for 1 hour; dead in 2 minutes.

Rabbit 8, 1,870 gm., $1 \mathrm{cc}$ in ear vein; dead in 30 seconds.

Rabbits Injected with Guinea-Pig Lung.-Rabbit 9, $600 \mathrm{gm} ., 0.5 \mathrm{cc}$; slight dyspnea only. Had survived Berkefeld filtrate of rabbit lung. Developed paralysis of both hind legs next day.

Guinea-Pigs Injected reith Guinea-Pig Lung Extract.-We omit those in which we believe we failed to enter the vein in the foot. Guinea-pig 1, 250 gm., $0.5 \mathrm{cc}$; scratched nose. Few minutes later $1 \mathrm{cc}$; dyspnea, paralysis, no convulsions; dead in 15 minutes.

Guinea-pig 2, $320 \mathrm{gm}$., less than $0.5 \mathrm{cc}$; collapse, respirations 25 per 10 seconds, comatose on side, gasping; recovered 25 minutes later.

Guinea-pig 7, $250 \mathrm{gm}$., injected with $1 \mathrm{cc}$; dyspnea, convulsions in $1.5 \mathrm{~min}-$ utes; dead in 6 minutes.

Guinea-pig 10, $250 \mathrm{gm} ., 1 \mathrm{cc}$; fell over in 1 minute, respirations 25 per 10 seconds, in 4, gasping in 9, and dead in 10 minutes.

Guinea-pig 11, $250 \mathrm{gm} ., 1 \mathrm{cc}$; dyspnea in 2 minutes; recovered.

A guinea-pig, which had been inoculated about a year previously from a guinea-pig dying of pernicious anemia, died of pneumonic consolidation of the lungs. No bacteria were found microscopically nor in cultures. Standard extract was made from this pneumonic lung and used as follows:

Guinea-pig 13, $300 \mathrm{gm} ., 0.5 \mathrm{cc}$; fell over in dyspnea in 20 seconds, rapid running motions with legs, occasional gasp, and death in 5 minutes.

Guinea-pig 14, $250 \mathrm{gm} ., 0.3 \mathrm{cc}$; dyspnea in 30 seconds, lying on side and passive 17 minutes later; recovered.

Guinea-pig 15, $300 \mathrm{gm} ., 0.4 \mathrm{cc}$; behaved like 13 ; died in 10 minutes.

Guinea-Pigs Injected with Rabbit Lung Extract.-Guinea-pig 3, $300 \mathrm{gm}$., $0.5 \mathrm{cc}$ into tissues of foot and then $0.75 \mathrm{cc}$ intravenously; weakness and dyspnea in 1 minute. Marked inspiratory dyspnea, and death in 3 minutes.

Giuinea-pig 5, $800 \mathrm{gm} ., 3.5 \mathrm{cc}$ intracardially; dyspnea and slight weakness only; recovered.

Negative Experiments with Similar Extracts of Liver, Kidney, Ileum and Spleen.-Rabbit liver extract made up like the lung extract, but an opaque, murky red fluid. Injected Rabbit $11,1,230 \mathrm{gm}$., with $1 \mathrm{cc}$ intravenously; no symptoms. This rabbit had been injected the day before with $1 \mathrm{c} c$ of rabbit. plasma which had been in contact with washed lung tissue sediment for 30 minutes and had shown no symptoms. The day after the liver extract inoculation it received $1 \mathrm{cc}$ of lung extract diluted with $9 \mathrm{cc} \mathrm{NaCl}$ solution and died in 30 seconds.

Rabbit kidney extract in the form of a murky red fluid was injected into a vein of Rabbit $\mathrm{j}, 800 \mathrm{gm}$., in $0.5 \mathrm{cc}$ dose without symptoms. Two days later it was injected with lung extract and died at once. Again a kidney extract was made up by weight and $10 \mathrm{cc}$ of the extract in the form of a murky red fluid was inoculated intravenously into Rabbit 13, 2,030 gm.; no symptoms.

A few minutes later, Rabbit 13 was inoculated with a similar extract of the lower ileum of a rabbit; no symptoms. One hour after the last injection, Rabbit 13 received intravenously $1 \mathrm{cc}$ of standard lung extract diluted to $10 \mathrm{cc}$ with $0.9 \% \mathrm{NaCl}$ solution; no symptoms at once, in 2 minutes dyspnea; in 3 minutes respirations were 38 per 10 seconds, in 8 minutes 41 , in 12 minutes 44 , and soon dropped to 20 . Survived. 
Whole rabbit spleen ground up with $10 \mathrm{cc}$ salt solution; $1 \mathrm{cc}$ intravenously into Rabbit 19, 1,350 gm.; no symptoms.

Experiments Showing that the Lethal Agent Does Not Pass the Berkefeld Candle $N$, and Is Removed by Animal Charcoal.--The standard rabbit lung extract was centrifuged at $1,200-1,500$ revolutions until clear. Some of this was filtered through a thoroughly washed Berkefeld candle $\mathrm{N}$ which was known to be proof to $B$. prodigiosus. The filtrate was perfectly clear but red with hemoglobin.

Rabbit 7, 1,666 gm., injected intravenously with $1 \mathrm{cc}$; no symptoms. Kept under observation for a week when it was used as control (33) when it succumbed at once to the unfiltered extract.

Rabbit 9, $600 \mathrm{gm}$., injected with $2 \mathrm{cc}$ of the filtrate; no symptoms. On the 4 th day thereafter it developed paralysis of both hind legs.

Control Rabbit $8,1,870 \mathrm{gm}$., injected with $1 \mathrm{cc}$ of the unfiltered extract; dead in 30 seconds.

A 2,000 gm, rabbit was exsanguinated and the lungs washed out through the right ventricle with $0.9 \%$ salt solution. The very pale lungs were cut to pieces and these kneaded out in salt solution, dried with filter paper and the pale washed substance ground up to make standard extract which was centrifuged. The supernatant fluid was clear and straw colored.

Rabbit $27,870 \mathrm{gm}$., injected intravenously with $0.3 \mathrm{cc}$ of this extract; convtlsions in 20 seconds, and dead in 45 seconds.

Rabbit 28, $890 \mathrm{gm}$; ; the extract was passed through a Berkefeld $\mathrm{N}$ at 600 mm. pressure; filtered very slowly; injected $0.3 \mathrm{cc}$ intravenously; no symptoms.

Rabbit $29,830 \mathrm{gm}$. ; the deposit on the outer surface of the filter used in the previous experiment was washed off with $3 \mathrm{cc}$ of salt solution; a cloudy fluid resembling ovomucoid was obtained; injected $0.3 \mathrm{cc}$ intravenously; dead in one minute; slight convulsions.

Rabbit 30, $650 \mathrm{gm}$; 住 $4 \mathrm{cc}$ and centrifuged at 1,200-1,500 revolutions for 20 minutes. The supernatant fluid was a perfectly clear, slightly opalescent fluid; injected $0.3 \mathrm{cc}$ intravenously; no symptoms.

Rabbit $32,1,200 \mathrm{gm}$., injected as Rabbit 30 , but with $1 \mathrm{cc}$; convulsions in 80 seconds, and dead in 110 seconds.

Rabbit $31,800 \mathrm{gm}$; the unfiltered portion of the extract poured off from the Berkefeld filter, was used; injected $0.3 \mathrm{cc}$; convulsions and death in 30 seconds.

Rabbit $33,1,660 \mathrm{gm}$., received $0.5 \mathrm{cc}$ of standard lung extract intravenously; dead in 1.5 minutes.

The same lung extract was filtered through a layer of animal charcoal on hard filter paper. The filtrate looked like a solution of soap in water. Rabbit 34, 1,350 gm., received $0.5 \mathrm{cc}$ intravenously. No symptoms. The filtrate was then centrifuged at 1,200-1,500 revolutions for 25 minutes; there was a moderate amount of sediment and opalescent fluid above. The sediment was mixed with $0.5 \mathrm{cc}$ salt solution and injected intravenously in Rabbit 35, $1,300 \mathrm{gm}$.; marked inspiratory dyspnea, paralysis, nystagmus and convulsions with cry; dead in one minute.

The suspension of sediment which killed Rabbit 35 was examined and showed numerous flocculent masses varying from 2-12 microns in diameter and apparently made up of smaller visible particles. In addition there were quite a number of bacteria which apparently came from the animal charcoal. The supernatant fluid showed the same flocculi but smaller in size. 
Standard extract of rabbit lung was made by grinding with sand and then with animal charcoal, which was added before the salt solution; the charcoal was centrifuged out; supernatant fluid clear like water; injected $1,200 \mathrm{gm}$. rabbit intravenously with $7 \mathrm{cc}$; no symptoms.

The Effect of Heat.-The crude rabbit lung extract was heated at $57 \mathrm{C}$. for 20 minutes; injected $2,000 \mathrm{gm}$. rabbit intravenously with $1 \mathrm{cc}$; died at once. Extract was boiled for one minute; coagulum centrifuged out at low speed; $2 \mathrm{cc}$ of the slightly opalescent supernatant fluid was injected intravenously into a $800 \mathrm{gm}$. rabbit; in one minute it sneezed and respirations became accelerated. No other symptoms.

Tolerance Produced by Rapidly Repeated Inoculations or by Dilute Extracts. -1 . Rabbit $\mathrm{j}, 800 \mathrm{gm}$., received $0.5 \mathrm{c} \mathrm{c}$ of a rabbit kidney extract intravenously without symptoms. Then it received rabbit lung extract intravenously as follows: $1 / 6 \mathrm{cc}$ at $1-2$ minute intervals until it had received $1 \mathrm{cc}$; no symptoms. A few minutes later, $0.5 \mathrm{cc}$ intravenously; no symptoms. But this tolerance did not last, for 24 hours later when it received $1 \mathrm{cc}$ of standard extract intravenously it died at once.

2. Rabbit h, 2,000 gm; rabbit lung tissue sediment was washed 3 times with salt solution; third supernatant fluid was injected as follows: $1 \mathrm{cc}, 1.5 \mathrm{cc}$, $2 \mathrm{cc}$ at minute intervals. No symptoms. Four days later it was inoculated intravenously with $1 \mathrm{cc}$ of standard rabbit lung extract; it did not show symptoms until one minute when its respirations were accelerated; in 2 minutes it could barely stand $u p$; it was paralyzed in 5 , went into convulsions in 6 , and died in 7 minutes.

Control Rabbit 3, $850 \mathrm{gm}$., received $0.5 \mathrm{cc}$ of the same lung extract; dead in one minute without convulsions.

3. Standard rabbit lung extract was centrifuged at 1,200-1,500 revolutions for one hour. The supernatant fluid, clear and translucent, was pipetted off $=\mathrm{A}$. The sediment was mixed with $4 \mathrm{cc}$ of $0.9 \% \mathrm{NaCl}$ solution and allowed to stand at room temperature for one hour; the supernatant fluid on this $=\mathrm{B}$.

Rabbit 5, 1,960 gm., received intravenously $1 \mathrm{cc}$ of $\mathrm{B}$; no symptoms. Then $2 \mathrm{cc}$ of $\mathrm{B}$; no symptoms. Then $2 \mathrm{cc}$ of $\mathrm{A}$; no symptoms. The last inoculation would have killed a normal rabbit, as shown by Rabbit 6 .

Control Rabbit 6, $1,960 \mathrm{gm}$., received intravenously $1 \mathrm{cc}$ of $\mathrm{A}$; dead in 2 minutes.

A lung sediment extract like that which gave protection to Rabbit 5 was centrifuged and the clear supernatant fluid tested for its protective action.

Rabbit 10, 1,500 gm., received $1 \mathrm{c} c$ intravenously; no symptoms. Then it received $1 \mathrm{c} c$ of standard lung extract; died in one minute.

Apparently something remained suspended in Extract B which stood for one hour that was capable of giving protection, but this was removed by centrifuging at high speed. This requires further confirmation.

Partial Protection Produced by Blood of a Shocked Rabbit.-Rabbit 25, $1,930 \mathrm{gm}$., received $0.3 \mathrm{c} \mathrm{c}$ standard rabbit lung extract. Dead in 2 minutes. Heart still beating 10 minutes later and blood not coagulated. The blood was allowed to flow into the thoracic cavity and $10 \mathrm{cc}$ was injected into the ear vein of Rabbit $26,1,660 \mathrm{gm}$. No reaction. About 10 minutes later injected $0.3 \mathrm{cc}$ standard lung extract. No symptoms for 5 minutes, but it fell over in 6 and died in 9 minutes. 
Incubation of Lung Extract with Homologous Corpuscles Does Not Increase Its Toxicity.-Made up standard lung extract from rabbit lung kept for 19 hours on ice $=\mathrm{A}$.

Incubated some of $\mathrm{A}$ in water bath at $40 \mathrm{C}$. for 15 minutees $=\mathrm{B}$.

Washed $1 \mathrm{cc}$ rabbit corpuscles two times with salt solution; suspended in $3 \mathrm{c} \mathrm{c}$ of $\mathrm{A}$ and heated as $\mathrm{B}=\mathrm{C}$.

Rabbit $20,1,685 \mathrm{gm}$., received $0.5 \mathrm{c} \mathrm{c}$ of $\mathrm{B}$; convulsions in 20 seconds, down in 30 , respirations 36 per 10 seconds; fine tremor over body in one minute; dead in 2 minutes.

Rabbit $21,1,715 \mathrm{gm}$., received intravenously $0.3 \mathrm{cc}$ of $\mathrm{B}$; convulsions in 30 , dead in 40 seconds.

Rabbit $22,1,670 \mathrm{gm}$., received intravenously $0.1 \mathrm{cc}$ of $\mathrm{B}$; acceleration of respirations in one minute to 39 per 10 seconds. No other symptoms.

Rabbit $24,1,880 \mathrm{gm}$., received intravenously $0.1 \mathrm{cc}$ of $\mathrm{C}$. No symptoms.

The Lethal Agent Is Not Removed by Ether-Took $4 \mathrm{cc}$ of standard rabbit lung extract and shook thoroughly with about $4 \mathrm{cc}$ ether in separatory funnel, The extracted filtrate, still containing some ether, was injected in $0.5 \mathrm{cc}$ dose intravenously in Rabbit 14, $700 \mathrm{gm}$. Dead in 30 seconds with marked convulsions and cries.

This extracted filtrate was kept at $5 \mathrm{C}$. over night and then placed in a vacuum to remove all the ether. When $0.5 \mathrm{cc}$ was injected intravenously in Rabbit 15, $700 \mathrm{gm}$., it produced little effect. In 6 seconds the respirations went to 38 per 10 seconds but very shortly fell to normal.

The light amber colored jelly removed by the ether was dried, redissolved in $4 \mathrm{cc} \mathrm{NaCl}$ solution and centrifuged clear. Rabbit $16,700 \mathrm{gm}$., received $0.5 \mathrm{cc}$ intravenously; no symptoms.

The Lethal Agent Can Be Removed by Centrifugal Force.-Made up standard rabbit lung extract from perfectly fresh lung. This was centrifuged at 3,000 revolutions for 10 minutes $=\mathrm{A}$.

The supernatant fluid A was recentrifuged in a special type of separator at 36,000 revolutions per minute for 15 minutes. This last supernatant fluid was perfectly clear but tinged with hemoglobin $=\mathrm{B}$.

The fine sediment on the sides of the separator was washed off with $0.9 \%$ $\mathrm{NaCl}$ solution $=\mathrm{C}$.

Rabbit 36, $1,190 \mathrm{gm} ., 0.3 \mathrm{cc}$ of $\mathrm{A}$ intravenously; respirations accelerated; survived.

Rabbit $37,1,080 \mathrm{gm} ., 0.5 \mathrm{cc}$ of $\mathrm{A}$; as 36 .

Rabbit $38,820 \mathrm{gm} ., 1 \mathrm{cc}$ of $\mathrm{A}$; dead in 2 minutes.

Rabbit 39, $800 \mathrm{gm} ., 2$ c c of B; no symptoms.

Rabbit $40,1,020 \mathrm{gm} ., 9 \mathrm{cc}$ of $\mathrm{B}$; dead in 1 minute.

Rabbit 36 was reinjected 15 minutes after the first inoculation with $0.3 \mathrm{cc}$ of a mixture of equal parts of $B$ and $C$; it scratched its nose and feet in one minute but showed no other symptom.

It is evident from these results that centrifuging at 3,000 revolutions per minute reduces the lethal action of the extract to about one-half that present when the centrifuging is performed at 1,200 or 1,500 , and that 36,000 revolutions per minute brings about a still further reduction. The fine particles which are thrown out are either the lethal agents themselves or adsorb the lethal agent. Microscopic examination of the clear fluid, obtained at 36,000 revolutions, showed the presence of scattered isolated particles about 0.5 microns (estimated) in diameter and small aggregations of a dozen or so of these; the sediment was composed of isolated and massed bodies like those 
described and of larger spherical refractile bodies 1.5-2 microns in diameter (estimated) and of aggregations of particles of both types. These granules did not stain well with Loeffler's methylene blue but stained with carbol gentian violet. Many of the large and some of the smaller particles gave a fat reaction with sudan III. None were acid-fast when stained by the tubercle method.

Morphin and Atropin Do Not Lessen the Lethal Action.-1. Rabbit 36, $1,190 \mathrm{gm} ., 0.08 \mathrm{gm}$. atropin sulphate slowly intravenously; then $0.5 \mathrm{cc}$ lung extract; fell over in 15 seconds, dead in 30 seconds. Marked tremor of muscles.

Rabbit 37, $1,080 \mathrm{gm}, 100 \mathrm{mg}$. atropin sulphate slowly intravenously and one minute later $0.5 \mathrm{c} \mathrm{c}$ lung extract. Dead in one minute. Symptoms typical.

2. Rabbit $41,1,500 \mathrm{gm} ., 1 / 4$ grain $(0.016 \mathrm{gm}$.) morphin sulphate intravenously and when lying passive it received $0.5 \mathrm{cc}$ lung extract. Dead in one minute, convulsions after rolling on side.

\section{SUM MARY}

An extract of finely ground rabbit or guinea-pig lung in $0.9 \% \mathrm{NaCl}$ solution is fatal for these animals when injected intravenously in a suitable dose. Rabbit lung is more fatal for the rabbit than for the guinea-pig and vice versa. Many of the symptoms of anaphylactic shock are produced-the acceleration of the respiration and peripheral irritation, both best shown by sublethal doses, the inspiratory dyspnea, the convulsions, the tolerance produced by graded repeated doses, the delay in the coagulation of the blood, all make one think of a possible relationship to anaphylaxis, but the lungs collapse on opening the thorax. Atropin does not protect. Morphin does not protect. Similar extracts of rabbit liver, kidney, ileum and spleen were harmless for rabbits.*

The lethal agent will not pass Berkefeld filter $\mathrm{N}$ at $600 \mathrm{~mm}$. pressure. It is removed by animal charcoal. It is not extracted by ether. It can be removed by centrifugal force (36,000 revolutions per minute) and is apparently composed of, or adsorbed to, particles visible under the microscope.

By beginning with a sublethal dose, and then injecting at short intervals thereafter larger doses, a rabbit will survive, without symptoms, several lethal doses, just as in Besredka's and Friedberger's methods of producing "antianaphylaxis."

\footnotetext{
* Since we are unable to continue this work at present we wish to add some further observations to the proof of this article. Lung tissue may be autoclaved at 15-20 pounds pressure for 15 minutes and then ground up and extracted as usual without appreciably altering its lethal action. Boiling the extract itself renders it innocuous. Next to lung tissue we have found omentum most toxic. Kidney extract will kill in a dose of 2 c.c. Muscle and brain extracts produced no symptoms in doses up to 2 c c. An inoculation of muscle or brain extract given about an hour before a fatal dose of lung or omentum extract protected the rabbits. The toxicity of normal and pneumonic human lung extracts for some rodents and dogs has been reported upon by Rosenow; Solis-Cohen, Weiss and Kolmer (Jour. Inf. Dis., 1918, 22, p. 476), but from a somewhat different viewpoint.
} 
It does not seem likely that the lethal agent comes from the interaction of lung tissue and homologous red blood cells, for incubating these together for a short time does not increase the poisonous action. Washed lung and thoroughly washed lung tissue fragments when ground up still yield particles which produce the symptoms and give protection. These particles can be removed by centrifugal force. A very similar toxic action was obtained by DeKruif ${ }^{1}$ in repeating earlier work on the toxicity of homologous normal serum obtained by defibrination. The work of F. G. Novy and his pupils and the still more recent work of Kritchewsky ${ }^{2}$ seems to throw the problem of anaphylactic shock into the field of colloid chemistry. While we are not entirely convinced that we are dealing with a particle phenomenon, we are inclined to favor the idea that colloidal particles are responsible for the lethal action. It still remains to be proven whether these particles have adsorbed to them something like an "internal secretion" capable of stimulating respiration.

1 Jour. Infect. Dis., 1917, 20, p. 717.

2 Ibid., 1918, 22, p. 101. 